\title{
Comprehensive Marine Particle Analysis System
}

\author{
Dr. Thomas Hopkins \\ Department of Marine Science \\ University of South Florida \\ St.Petersburg, FL 33701-5016 \\ CO-PIs: Lawrence Langebrake, Tracey Sutton \\ phone: (727) 553-1501 fax: (727) 553-3967 email: thopkins@marine.usf.edu \\ Award \#: N000149615020
}

\section{LONG-TERM GOAL}

The long term goal of this project is the development and utilization of a comprehensive (broadly capable) marine particle analysis system. The system is designed with wide dynamic range, thus, it will ultimately be used for high speed, high resolution characterization of water column particle fields in high, medium and low latitudes. As part of a broader goal this project continues advancement of the AOSN concept through joint use of both the towed platform and autonomous underwater vehicles.

\section{OBJECTIVES}

The project's objective is to develop a comprehensive marine particle analysis system which, along with other sensor systems, will enable us to address basic oceanographic, environmental and ultimately military issues. The objective includes adapting sensors to towed platforms and AUVs to characterize particle fields in a variety of oceanic environments.

\section{APPROACH}

The high resolution sampler (HRS) is designed to measure ocean properties that will provide insight into water column chemical, physical and biological processes. The platform has been used in Gulf of Mexico deployments to gather meaningful data and show efficacy. The approach for this project is to complete the sensor prototypes initiated in the period 1994 to early 1998; test them at the USF Center for Ocean Technology (COT); sea-test them on the HRS towed platform; and then adapt sensors, as available, to Florida Atlantic University AUVs. Deployments of the HRS in the Gulf of Mexico and deployments of an AUV in the South Florida Test Facility are part of this project. Acquired data will allow researchers access to an enhanced data set for improvement and advancement of their models.

\section{WORK COMPLETED}

\section{A. Sensor Development}

\section{i. Shadowed Image Particle Profiling and Evaluation Recorder (SIPPER)}

This instrument images marine particles in situ by recording the silhouettes of any particles in its optical light path. The instrument and supporting software were completed during this reporting period, and deployed on several occasions. The system consists of a pair of low-power laser line generators, 


\section{Report Documentation Page}

Form Approved

OMB No. 0704-0188

Public reporting burden for the collection of information is estimated to average 1 hour per response, including the time for reviewing instructions, searching existing data sources, gathering and maintaining the data needed, and completing and reviewing the collection of information Send comments regarding this burden estimate or any other aspect of this collection of information,

including suggestions for reducing this burden, to Washington Headquarters Services, Directorate for Information Operations and Reports, 1215 Jefferson Davis Highway, Suite 1204, Arlington

VA 22202-4302 Respondents should be aware that notwithstanding any other provision of law, no person shall be subject to a penalty for failing to comply with a collection of information if it

does not display a currently valid OMB control number

\begin{tabular}{|c|c|c|}
\hline $\begin{array}{l}\text { 1. REPORT DATE } \\
\text { 30 SEP } 1999\end{array}$ & 2. REPORT TYPE & $\begin{array}{l}\text { 3. DATES COVERED } \\
\mathbf{0 0 - 0 0 - 1 9 9 9} \text { to 00-00-1999 }\end{array}$ \\
\hline \multirow{3}{*}{\multicolumn{2}{|c|}{$\begin{array}{l}\text { 4. TITLE AND SUBTITLE } \\
\text { Comprehensive Marine Particle Analysis System }\end{array}$}} & 5a. CONTRACT NUMBER \\
\hline & & 5b. GRANT NUMBER \\
\hline & & 5c. PROGRAM ELEMENT NUMBER \\
\hline \multirow{3}{*}{\multicolumn{2}{|c|}{ 6. AUTHOR(S) }} & 5d. PROJECT NUMBER \\
\hline & & 5e. TASK NUMBER \\
\hline & & 5f. WORK UNIT NUMBER \\
\hline \multicolumn{2}{|c|}{$\begin{array}{l}\text { 7. PERFORMING ORGANIZATION NAME(S) AND ADDRESS(ES) } \\
\text { University of South Florida,Department of Marine Science,140 Seventh } \\
\text { Ave. South,St. Petersburg,FL,33701 }\end{array}$} & $\begin{array}{l}\text { 8. PERFORMING ORGANIZATION } \\
\text { REPORT NUMBER }\end{array}$ \\
\hline \multirow{2}{*}{\multicolumn{2}{|c|}{ 9. SPONSORING/MONITORING AGENCY NAME(S) AND ADDRESS(ES) }} & 10. SPONSOR/MONITOR'S ACRONYM(S) \\
\hline & & $\begin{array}{l}\text { 11. SPONSOR/MONITOR'S REPORT } \\
\text { NUMBER(S) }\end{array}$ \\
\hline
\end{tabular}

12. DISTRIBUTION/AVAILABILITY STATEMENT

Approved for public release; distribution unlimited

13. SUPPLEMENTARY NOTES

14. ABSTRACT

15. SUBJECT TERMS

16. SECURITY CLASSIFICATION OF:

\begin{tabular}{c|c|c}
$\begin{array}{c}\text { a REPORT } \\
\text { unclassified }\end{array}$ & $\begin{array}{c}\text { b ABSTRACT } \\
\text { unclassified }\end{array}$ & $\begin{array}{c}\text { c THIS PAGE } \\
\text { unclassified }\end{array}$
\end{tabular}

17. LIMITATION OF ABSTRACT

Same as

Report (SAR)
18. NUMBER 19a. NAME OF

OF PAGES RESPONSIBLE PERSON

7

Standard Form 298 (Rev. 8-98) Prescribed by ANSI Std Z39-18 
collimating and imaging optics, and digital imaging and data storage electronics. SIPPER can generate up to 80 minutes of high-resolution $(50 \times 50 \mu \mathrm{m}$ at $1 \mathrm{~m} / \mathrm{s})$ gap-free images from two orthogonal directions over a large active area $(96 \times 96 \mathrm{~mm})$. Real-time feedback and sensor control is provided via a LON network. A photo of SIPPER mounted in a Florida Atlantic University OEX AUV is shown in Fig. 1.

After recording images, binary data is compressed and sent via a 10Mbps Ethernet connection to a shipboard computer. Images may be reproduced on-screen immediately with custom software (SIPPERView), and/or archived. SIPPERView quickly sifts through data and generates a list of significant particles. The user can view, identify and size the specimens. The SIPPERView application is shown in Fig. 2. A more detailed description of the instrument and software may be found in [3].

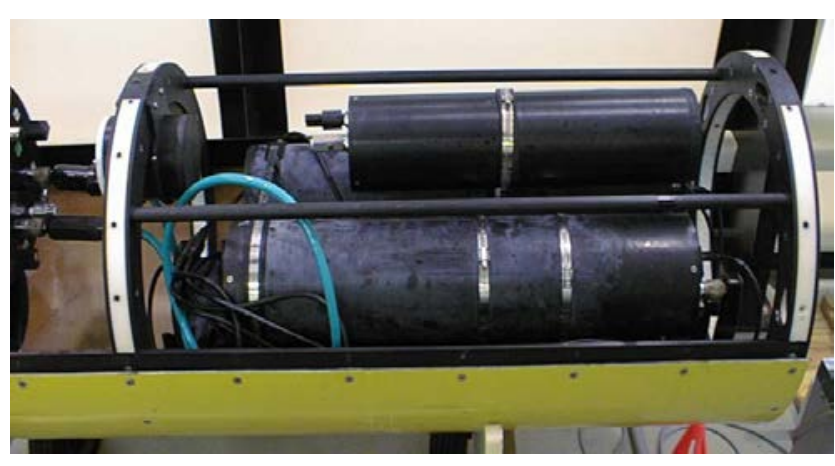

1. SIPPER instrument mounted in AUV nosecone. The SIPPER data storage vessel is located fore (connectors shown at left) and imaging and battery tubes located aft. Dual Light Sheet instrument (see Section iv) is mounted aft of data storage vessel for deployments.

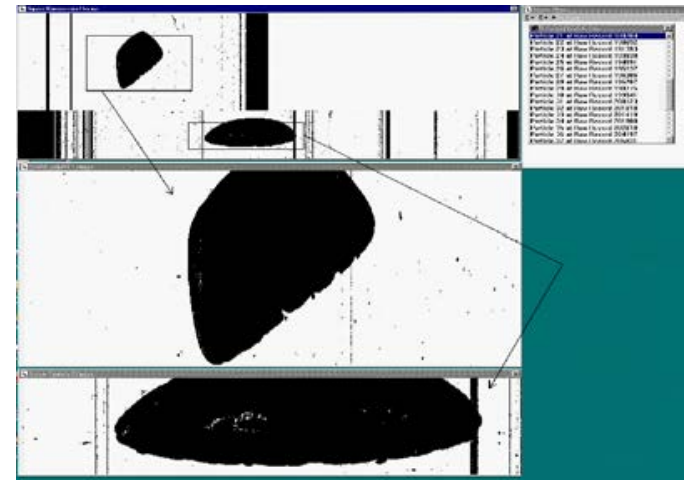

2. SIPPERView software showing from upper right corner: particle selection window, low-resolution view of a thimble jellyfish (full $96 \times 96 \mathrm{~mm}$ ), camera 1 (2048 pixels) magnified, camera 2 (4096 pixels) magnified. Camera 2 images not to scale. Data from COBOP (Lee Stocking Island) June 1999.

\section{ii. Particle Image Recorder (PIR)}

This instrument is designed to video-record marine particles as they enter the towed platform main sample port. Two modes of operation have been designed into this system: 1) strobe "still frame" mode and; 2) Continuous wave (CW) mode where a tungsten lamp illuminates the sample volume continuously. Mode 2 allows determination of particle movement with respect to the towed platform by streak pattern analysis. This system is under construction and is expected complete in the near future.

\section{iii. Chirp Bio-scatter Sonar}

Work is continuing on packaging the unit for deployment on the HRS and OEX platforms. Additionally, schematics for the system are being converted into OrCAD format to allow for rapid manufacture and testing of the entire unit. Efforts are underway with Gage Applied Sciences and Mathworks to integrate the Gage D/A board with MatLab software allowing for more rapid implementation of large FFT processing of the acoustic data. Transducers for the unit have been purchased and tested successfully during this period. 


\section{iv. Dual Light Sheet (DLS)}

This sensor was completed in late 1997 with AUV testing in June 1998 and HRS testing in July and September 1998. Additional deployments were completed in March, June, and July 1999. Enhancements were made under this year's project to improve small particle sensitivity and ease calibration. Additional work towards an entirely new DLS unit was completed. Conceptual design, electronics, and base line software have were completed with testing expected by the end of the calendar year.

The DLS velocity measurement system was integrated in prior year's project was tested both on the HRS and AUV. HRS and AUV testing showed excellent results. Testing on the HRS showed significant reduction in sample flow rate with a sample net in place versus no-net. DLS correlation software uses a cross correlation algorithm to calculate particle velocity with approximately $2 \%$ accuracy. Data from a June 1999 HRS test deployment is shown in Fig. 3.

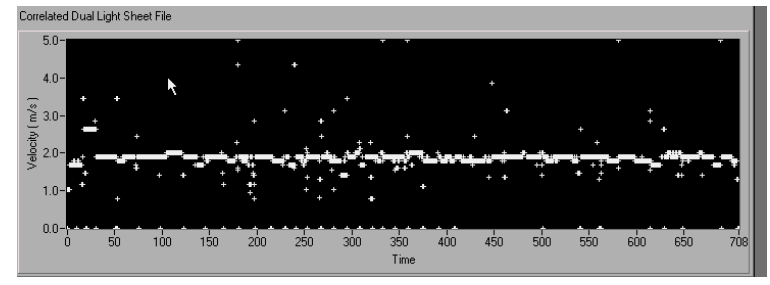

a)

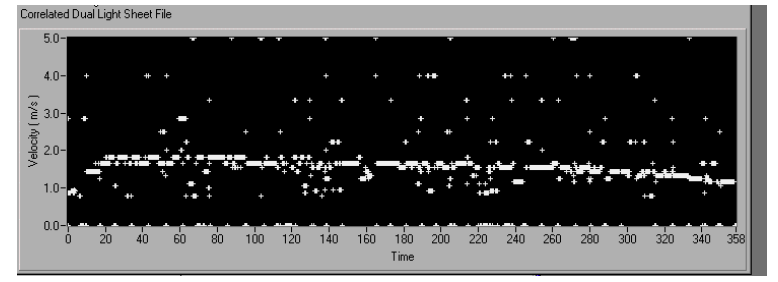

b)

3 a) HRS sample tube water velocity versus time (no net). b) HRS sample tube water velocity versus time (160 $\mu$ net installed). Note decrease in sample tube velocity for net versus no-net at start of run and gradual decrease in sample tube water velocity for net installed case over time. Platform tow velocity is the same for both cases.

\section{B. Platform Development}

\section{i. $H R S$}

Further testing and refinement of the Niskin bottle array has resulted in a reliable system for obtaining water samples for small particle, chlorophyll and nutrient analyses. This system has become a key component in estimating bio-optical coupling at the HyCODE/ECOHAB: Florida test site. Preliminary data from this site has emphasized the importance of the small zooplankton size fraction $(<1 \mathrm{~mm}$ forms dominant numerically). To sample this size fraction, a series of $80 \mu$ nets were designed, constructed and tested. Additional protocols have been established for foul-weather shipboard handling (deployment, retrieval), with the new method allowing deployments in seas up to 8 '.

\section{ii. HRS System Improvements}

Data acquisition has been shifted from desktop computers to laptop/docking station units, greatly reducing shipboard bench-top space requirements. A Chelsea Instruments fast repetition rate fluorometer (FRRF) has been integrated into the HRS system. This instrument, though still in its infancy, has the potential to allow in situ estimates of primary production, which can then be correlated 
to other measured variables. Syntactic foam forms were added to the HRS to balance this payload for proper towing attitude.

\section{iii. Dual Light Sheet - SIPPER AUV integration}

The Dual Light Sheet - SIPPER AUV integration involved construction of an entirely new OEX payload section. The payload section was custom fitted using Pro-E cad software before pressure vessels were fabricated to ensure fit (see Fig. 1). The integration required extensive outer shell modification with a large portion of the main computer pressure vessel protruding from the front of the upper half shell. Despite the alterations, the OEX vehicle flight characteristics were not significantly altered and deployments were completed smoothly and without incident.

\section{HRS and AUV Deployments}

\section{i. HRS deployments}

HRS testing and sampling was conducted in three locations in 1999: testing the FRRF configuration for trim and signal was conducted near the mouth of Tampa Bay (10 Jun); HRS sampling was conducted twice (27-31 Mar and 17-19 Jul) at the HyCODE/ECOHAB: Florida site; and sampling was conducted in the oceanic eastern Gulf of Mexico (20-29 Jul) to continue earlier water mass characterization at this site [3] (Fig. 4). At this site the SIPPER and DLS instruments were successfully used to a depth of 200 $\mathrm{m}$ for extended periods. Comparison of net-caught specimens with their SIPPER images (Fig. 5) illustrates the effectiveness of in situ particle sensing.

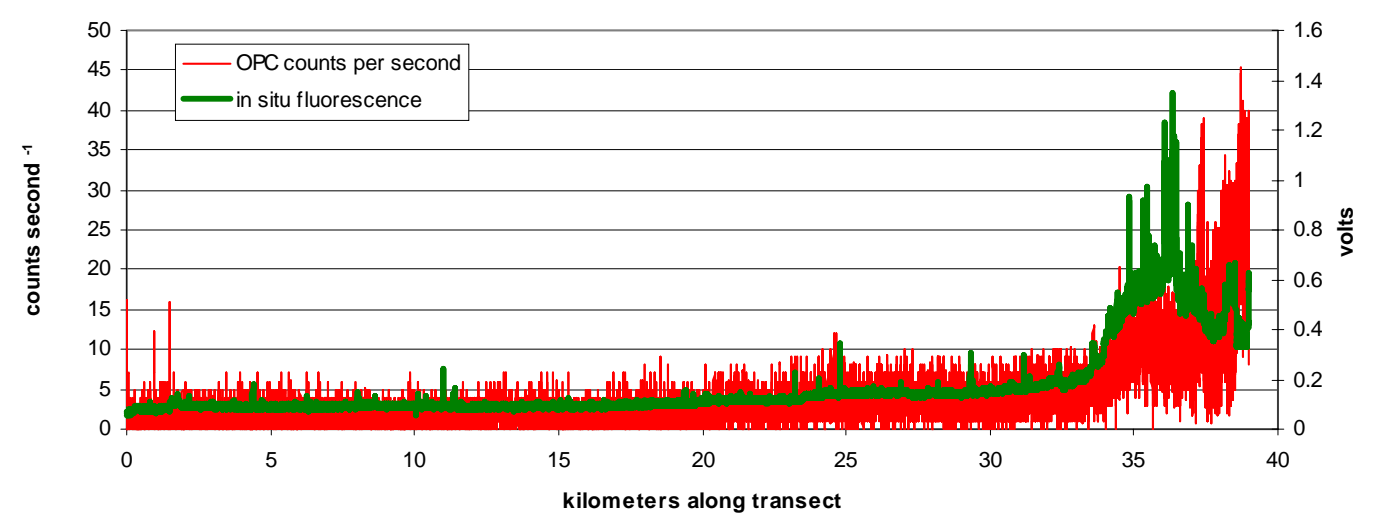

\section{Chlorophyll fluorescence and zooplankton-sized particle distributions across a water mass discontinuity at the oceanic eastern Gulf of Mexico site.}

\section{ii. AUV Deployments of DLS and SIPPER instruments}

Several deployments of the SIPPER and DLS were made in the Bahamas (CoBOP), and Ft. Lauderdale. Successful deployments were made on both towed (HRS) and AUV platforms. After successful testing on a recirculating flume, the SIPPER package was tested for the first time at sea in March 1999. After correcting a mechanical defect in one mirror within SIPPER, it was deployed with DLS on a Florida Atlantic AUV at CoBOP in May 1999. The digital particle locating software proved especially valuable in the sparse waters near Lee Stocking Island.. 
Fragile specimens (eg. siphonophores, jellyfish) were captured intact by SIPPER, while being fractured by physical net sampling.

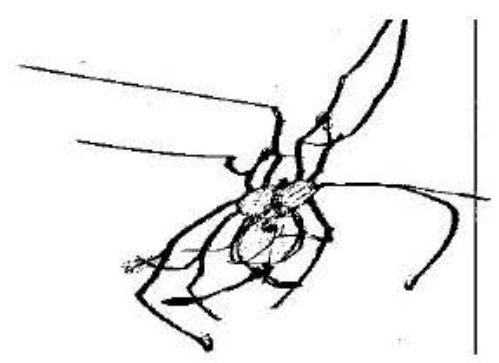

a) camera 1

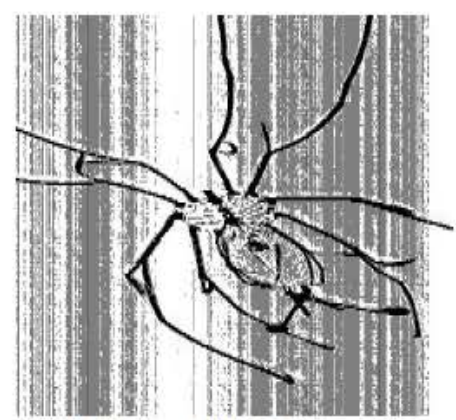

b) camera 2

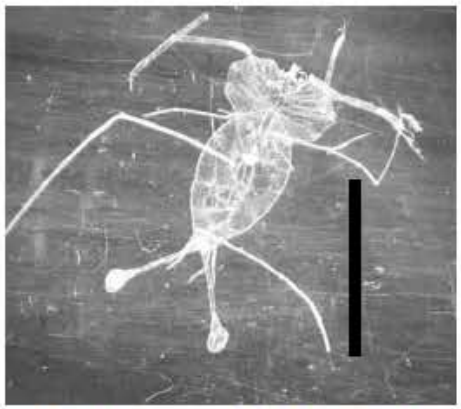

c) microscope (from net)

\section{SIPPER $(a, b)$ and microscope images $(c)$ of lobster phyllosoma sampled during July, 1999 HRS cruise. Marker bar in microscope image is $10 \mathrm{~mm}$.}

D. Other

i. Establishment of a laboratory photography/image analysis system to process samples collected with the HRS carousel plankton codends.

The current system, constructed by Tracey Sutton, consists of three components: 1) microscope with video camera adapter; 2) camera/monitor system; and 3) computer/software system. Video images of HRS samples are obtained via a Sony DXC-970MD 3CCD color video camera mounted on an Olympus SZH10 stereoscope. These video images are routed to a Sony Trinitron color monitor through a Sony Color Video Printer. The monitor outputs to an Integral Technologies FlashPoint PCI frame grabber board installed in a Compaq Deskpro 6000 computer. The images are then processed real-time via Optimas 6.5 image analysis software. The advantages of this system are: 1) particle measurement rate and accuracy are increased via mouse-driven cursor controls (vs. ocular micrometer methodology); 2) sample images can be archived on optical disk for further analysis; and, 3) data are downloaded real-time to Windows-based spreadsheet programs, thus reducing the time and human error of manual data entry.

\section{RESULTS}

-Data gathered from HRS II deployments provided information for the HyCODE / ECOHAB: Florida model and a manuscript has been submitted to a peer-reviewed journal based on these data. $\bullet$ An AUVmounted particle sensor (DLS) was tested. Data were retrieved showing excellent results including prove-out of a particle velocity measurement system. $\bullet$ Construction and testing of a high-resolution insitu particle imaging system (SIPPER) was completed, with integration of this system on both the HRS and AUV platforms. $\bullet$ A lab-based plankton analysis facility was created for ground-truthing of electronically acquired data. This will allow for identification of problems with developed instruments and ultimately improve electronically acquired data sets.. 


\section{IMPACT/APPLICATIONS}

This project represents a directed effort to build and test systems for characterization of a wide variety of marine environments. Data gathered have a direct application to predictive biological process models, particularly as they apply to optical properties of seawater in coastal ecosystems. The sensors being developed and tested are targeted for deployment on modern AUV's. Experience gained in deploying and developing sensors for AUV's will have significant impact on defining the appropriate tools for future automated monitoring of the ocean.

\section{TRANSITIONS}

The data output of this project will be of interest to programs such as HyCODE / ECOHAB. HRS data indicate that seawater optical properties at the HyCODE/ECOHAB: Florida site derive mainly from biotic activity (i.e. dissolved and particulate carbon) rather than sediment suspension processes (Fig. 6). Others involved in the optical properties of water, and those creating biological - chemical physical process-linked models will use the data

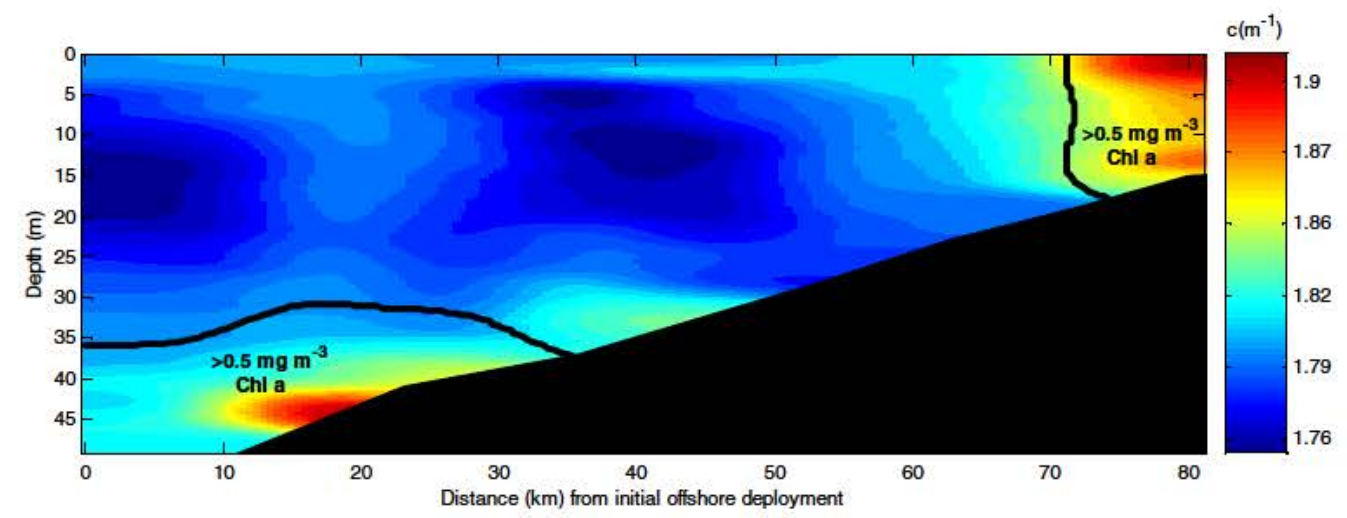

\section{Beam attenuation profile for $\mathrm{HyCODE/ECOHAB:} \mathrm{Florida} \mathrm{site} \mathrm{with} \mathrm{relatively}$ high chlorophyll $\left(>0.5 \mathrm{mg} \mathrm{m}^{-3}\right)$ regions contoured in black.}

\section{RELATED PROJECTS}

1) Enhanced in-situ spectroscopic analyses of trace seawater solutes.

2) Construction of in-situ underwater mass spectrometer .

\section{REFERENCES}

1) Davis, C.S., S.M. Gallager, M.S. Berman, L.R. Haury and J.R. Strickler. 1992. The video plankton recorder (VPR): Design and initials results. Arch. Hydrobiol./Beih. Ergebn. Limnol. 36: 67-81.

2) Herman, A.W. 1992. Design and calibration of a new optical plankton counter capable of sizing small zooplankton. Deep-Sea Res. 39: 395-415.

3) Remsen, A., T.L. Hopkins, T.T. Sutton. "Characterization of a low salinity feature at an oceanic site in the Gulf of Mexico". 1999 Meeting of the American Society of Limnologists and Oceanographers. Santa Fe, NM. 
4) Samson,S., L. Langebrake, C. Lembke and J. Patten. 1999 "Design and initial results of highresolution shadowed image particle profiling and evaluation recorder," Oceans '99 MTS/IEEE Conference Proceedings, v. 1, p. 58-63.

5) Sutton, T.T., T.L. Hopkins, A. Remsen, S. Burghart. Multisensor sampling of pelagic ecosystem variables in a coastal environment to estimate zooplankton grazing impact. Continental Shelf Research. Submitted. 